\title{
Formation of Enamides via Palladium(II)-Catalyzed Vinyl Transfer from Vinyl Ethers to Nitrogen Nucleophiles
}

\author{
Jodie L. Brice, James E. Meerdink, and Shannon S. Stahl* \\ University of Wisconsin - Madison \\ 1101 University Ave. \\ Madison, WI 53706 \\ Email: stahl@chem.wisc.edu
}

Supporting Information

\section{General Considerations.}

All commercially available compounds were used as received, and all were purchased from Aldrich except DIPHOS and $\mathrm{Pd}\left(\mathrm{OCOCF}_{3}\right)_{2}(\mathrm{Strem}), \operatorname{Pd}(\mathrm{OAc})_{2}$ and $\mathrm{PdCl}_{2}$ (DuPont), $\mathrm{HgSO}_{4}$ (Mallinckrodt Chemical Works), and $\mathrm{HgCl}_{2}$ (Allied Chemical).

${ }^{1} \mathrm{H},{ }^{13} \mathrm{C}$, and ${ }^{19} \mathrm{~F}$ NMR spectra were recorded on Bruker AC-300 $\mathrm{MHz}$ spectrometers, and $\mathrm{CDCl}_{3}$ was purchased from Cambridge Isotope Laboratories, Inc. The chemical shifts $(\delta)$ are given in parts per million relative to internal TMS $\left(0\right.$ ppm for $\left.{ }^{1} \mathrm{H}\right)$, $\mathrm{CDCl}_{3}$ (77.2 ppm for ${ }^{13} \mathrm{C}$ ), and internal (capillary) $\mathrm{CF}_{3} \mathrm{CO}_{2} \mathrm{H}\left(-76.5 \mathrm{ppm}\right.$ for $\left.{ }^{19} \mathrm{~F}\right)$.

Flash chromatography was performed on silica gel 60 (particle size 0.0400.063mm, 230-400 mesh ASTM, purchased from EMD) with hexanes/diethyl ether or methylene chloride.

(DPP)Pd( $\left(\mathrm{OCOCF}_{3}\right)_{2}$ was prepared by a literature procedure, ${ }^{1}$ and (DPP) $\mathrm{Pd}(\mathrm{OAc})_{2}$, $(\mathrm{Phen}) \mathrm{Pd}\left(\mathrm{OCOCF}_{3}\right)_{2}, \quad(\mathrm{TMEDA}) \mathrm{Pd}\left(\mathrm{OCOCF}_{3}\right)_{2}, \quad\left(\mathrm{PPh}_{3}\right)_{2} \mathrm{Pd}\left(\mathrm{OCOCF}_{3}\right)_{2}, \quad$ and (DIPHOS)Pd( $\left(\mathrm{OCOCF}_{3}\right)_{2}$ were prepared analogously. (DPP) $\mathrm{PdCl}_{2}$ was prepared by a known method. ${ }^{2}$ 


\section{General procedure for catalyst screening.}

To a disposable culture tube were added 2-oxazolidinone $(0.19 \mathrm{mmol}, 16.8 \mathrm{mg})$, catalyst $(9.5 \mu \mathrm{mol})$, and 1,3,5-tri-tert-butylbenzene $(0.063 \mathrm{mmol}, 15.8 \mathrm{mg})$ internal standard. The reaction was started by adding butyl vinyl ether (BVE) (1.93 mmol, 250 $\mu \mathrm{L})$ and immediately heating to $75^{\circ} \mathrm{C}$. The culture tube was left open to air, and the contents were vortexed for 3 minutes. The reactions were halted by removing BVE under vacuum and cooling to $-78{ }^{\circ} \mathrm{C}$ within 45 seconds. Product yield was evaluated by ${ }^{1} \mathrm{H}$ NMR spectroscopy relative to internal standard.

\section{Synthesis of N-tosyl-N-vinyl- $\beta$-alanine methyl ester.}

The amine functionality of $\beta$-alanine was tosylated with $\mathrm{TsCl}$ according to literature procedure, $48 \%$. ${ }^{3}$ Subsequent methylation ${ }^{4}$ to the ester by TMS-CHN 2 furnished quantitative yield of this substrate for transfer vinylation. Characterization data agrees with literature. ${ }^{5}$<smiles>COC(=O)CCNC(F)(F)F</smiles>

\section{Representative procedure for transfer vinylation.}

2-Oxazolidinone (5.7 mmol, $500 \mathrm{mg}$ ), BVE (57.4 mmol, $7.43 \mathrm{~mL}$ ), and $(\mathrm{DPP}) \mathrm{Pd}\left(\mathrm{OCOCF}_{3}\right)_{2}(0.28 \mathrm{mmol}, 189 \mathrm{mg})$ were combined in a round bottom flask equipped with a magnetic stir bar. The flask was capped by a rubber septum with an 18 gauge needle punctured through it. The reaction was stirred at $75{ }^{\circ} \mathrm{C}$ in an oil bath and monitored for completion by ${ }^{1} \mathrm{H}$ NMR aliquots. Upon completion, the reaction mixture was allowed to cool and loaded directly onto a chromatography column packed with silica (elution solvent $=$ hexanes/ether 1:9).

\section{Product Characterization Data.}

3-vinyl-2-oxazolidinone. 91\%, light yellow oil. Characterization data matches literature report. 6 
<smiles>C=CN1CCOC1=O</smiles>

(S)-4-isopropyl-3-vinyl-2-oxazolidinone. Column chromatography (hexanes/ether 25:75) yielded a off-white solid, 95\%. ${ }^{1} \mathrm{H}$ NMR $\left(300 \mathrm{MHz}, \mathrm{CDCl}_{3}\right) \delta 6.78(\mathrm{dd}, \mathrm{J}=16.2$, $9.3 \mathrm{~Hz}, 1 \mathrm{H}), \delta$ 4.49-4.08 (m, 4H), $\delta 4.05(\mathrm{dt}, \mathrm{J}=4.8,3.6 \mathrm{~Hz}, 1 \mathrm{H}), \delta 2.45$ (septet d, J = $6.9,3.3 \mathrm{~Hz}, 1 \mathrm{H}), \delta 0.94(\mathrm{~d}, \mathrm{~J}=6.9 \mathrm{~Hz}, 3 \mathrm{H}), \delta 0.85(\mathrm{~d}, \mathrm{~J}=6.6 \mathrm{~Hz}, 3 \mathrm{H}) .{ }^{13} \mathrm{C}\left\{{ }^{1} \mathrm{H}\right\} \mathrm{NMR}$ $\left(75 \mathrm{MHz}, \mathrm{CDCl}_{3}\right) \delta 155.8,129.0,94.2,63.1,58.1,26.0,18.0,14.0$. HRMS: $\mathrm{m} / \mathrm{z}$ (EI) calculated $[\mathrm{M}]^{\cdot+}=155.0946$, measured 155.0942. $[\alpha]_{\mathrm{D}}^{25}=+51.5\left(\mathrm{c}=1.1, \mathrm{CH}_{2} \mathrm{Cl}_{2}\right)$.<smiles>C=CN1C(=O)OCC1C(C)C</smiles>

(S)-4-benzyl-3-vinyl-2-oxazolidinone. Column chromatography (hexanes/ether 25:75) yielded a light yellow oil, 92\%. Characterization data matches literature report. ${ }^{7}$<smiles>C=CN1C(=O)OCC1Br</smiles>

1-Vinyl-2-pyrrolidinone. Column chromatography (hexanes/ether 1:9) yielded a pale yellow oil, 89\%. ${ }^{1} \mathrm{H}$ NMR spectra matches known reports for commercially available compound. ${ }^{8}$<smiles>C=CN1CCCC1=O</smiles>

N-methyl-N-vinyl-p-toluenesulfonamide. ${ }^{9}$ Column chromatography $\left(\mathrm{CH}_{2} \mathrm{Cl}_{2}\right)$ yielded a white crystalline solid, 64\%. ${ }^{1} \mathrm{H}$ NMR $\left(300 \mathrm{MHz} \mathrm{CDCl}_{3}\right) \delta 7.64(\mathrm{~d}, \mathrm{~J}=6.75 \mathrm{~Hz}, 2 \mathrm{H}), \delta$ $7.31(\mathrm{~d}, \mathrm{~J}=6.25 \mathrm{~Hz}, 2 \mathrm{H}), \delta 7.00(\mathrm{dd}, \mathrm{J}=15.8,9.0 \mathrm{~Hz}, 1 \mathrm{H}), \delta 4.33(\mathrm{~d}, \mathrm{~J}=9.0 \mathrm{~Hz}, 1 \mathrm{H}), \delta$ $4.18(\mathrm{~d}, \mathrm{~J}=15.5 \mathrm{Hx}, 1 \mathrm{H}), \delta 2.86(\mathrm{~s}, 3 \mathrm{H}), \delta 2.43(\mathrm{~s}, 3 \mathrm{H}) .{ }^{13} \mathrm{C}\left\{{ }^{1} \mathrm{H}\right\} \mathrm{NMR}\left(75 \mathrm{MHz}, \mathrm{CDCl}_{3}\right)$ 
$\delta$ 143.6, 134.6, 129.6, 126.8, 92.9, 31.1, 21.3. HRMS: m/z (ESI) calculated $[\mathrm{M}+\mathrm{Na}+\mathrm{MeOH}]^{+}=266.0827$, measured $=266.0835$.

MeNTs

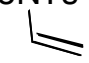

N-tosyl-N-vinyl- $\beta$-alanine methyl ester. Column chromatography $\left(\mathrm{CH}_{2} \mathrm{Cl}_{2}\right)$ yielded a pale yellow viscous oil, 87\%. ${ }^{1} \mathrm{H}$ NMR $\left(300 \mathrm{MHz}, \mathrm{CDCl}_{3}\right) \delta 7.67(\mathrm{~d}, \mathrm{~J}=6.6 \mathrm{~Hz}, 2 \mathrm{H}), \delta$ $7.31(\mathrm{~d}, \mathrm{~J}=8.7 \mathrm{~Hz}, 2 \mathrm{H}), \delta 6.88(\mathrm{dd}, \mathrm{J}=15.9,9.3 \mathrm{~Hz}, 1 \mathrm{H}), \delta 4.36(\mathrm{~d}, \mathrm{~J}=9.6 \mathrm{~Hz}, 1 \mathrm{H}), \delta$ $4.28(\mathrm{~d}, \mathrm{~J}=15.9 \mathrm{~Hz}, 1 \mathrm{H}), \delta 3.69(\mathrm{~s}, 3 \mathrm{H}), \delta 3.64(\mathrm{~m}, 2 \mathrm{H}), \delta 2.65(\mathrm{~m}, 2 \mathrm{H}), \delta 2.43(\mathrm{~s}, 3 \mathrm{H})$. ${ }^{13} \mathrm{C}\left\{{ }^{1} \mathrm{H}\right\}$ NMR $\left(75 \mathrm{MHz}, \mathrm{CDCl}_{3}\right) \delta 171.6,144.2,136.0,131.8,130.0,127.0,92.9,52.0$, 40.4, 32.0, 21.7. HRMS: $\mathrm{m} / \mathrm{z}(\mathrm{ESI})$ calculated $[\mathrm{MNa}]^{+}=306.0776$, measured $=306.0763$.<smiles>C=CN([13CH3])CCC(=O)OC</smiles>

N-vinyl-2,2,2,-trifluoroacetamide. ${ }^{10}$ Due to difficulties encountered during purification of N-vinyl trifluoroacetamide, ethyl vinyl ether was used in place of butyl vinyl ether and the reaction was run at room temperature. Column chromatography $\left(\mathrm{CH}_{2} \mathrm{Cl}_{2}\right)$ yielded a white solid, 76\%. ${ }^{1} \mathrm{H}$ NMR (300 MHz, $\mathrm{CDCl}_{3}$ ) $\delta 8.15$ (br., 1H), $\delta 6.89$ (ddd, J = 18.9, 15.6, $9.0 \mathrm{~Hz}, 1 \mathrm{H}), \delta 5.01(\mathrm{~d}, \mathrm{~J}=9.0 \mathrm{~Hz}, 1 \mathrm{H}), \delta 4.79(\mathrm{~d}, \mathrm{~J}=9.0 \mathrm{~Hz}, 1 \mathrm{H}) .{ }^{13} \mathrm{C}\left\{{ }^{1} \mathrm{H}\right\} \mathrm{NMR}$ $\left(75 \mathrm{MHz}, \mathrm{CDCl}_{3}\right) \delta 155.0$ (q, J = 38.2 Hz), $\delta 126.4(\mathrm{~s}), \delta 115.8$ (q, J = $\left.287 \mathrm{~Hz}\right), \delta 102.0$ (s). ${ }^{19} \mathrm{~F}$ NMR $\left(282 \mathrm{MHz}, \mathrm{CDCl}_{3}\right) \delta$ 74.7. HRMS: $\mathrm{m} / \mathrm{z}$ (EI) calculated $[\mathrm{M}]^{\circ+}=139.0245$, measured $=139.0247$.<smiles>C=CNC(=O)C(F)(F)F</smiles>

Incompatibility of the described transfer vinylation conditions with the carboxylic acid functionality.<smiles>C=CN([13CH3])CCC(=O)OC(C)OCCCC</smiles> 
Column chromatography $\left(\mathrm{CH}_{2} \mathrm{Cl}_{2}\right)$ yielded a pale yellow oil, $12 \%$. ${ }^{1} \mathrm{H}$ NMR (300 $\left.\mathrm{MHz}, \mathrm{CDCl}_{3}\right) \delta 7.67(\mathrm{~d}, \mathrm{~J}=6.9 \mathrm{~Hz}, 2 \mathrm{H}), \delta 7.31(\mathrm{~d}, \mathrm{~J}=7.8 \mathrm{~Hz}, 2 \mathrm{H}), \delta 6.88(\mathrm{dd}, \mathrm{J}=16.2$, $9.3 \mathrm{~Hz}, 1 \mathrm{H}), \delta 5.91(\mathrm{q}, \mathrm{J}=5.4 \mathrm{~Hz}, 1 \mathrm{H}), \delta 4.37$ (d, J = $9.0 \mathrm{~Hz}, 1 \mathrm{H}), \delta 4.28(\mathrm{~d}, \mathrm{~J}=15.6 \mathrm{~Hz}$, $1 \mathrm{H}), \delta 3.66-3.45(\mathrm{~m}, 4 \mathrm{H}), \delta 2.66(\mathrm{t}, \mathrm{J}=6.6 \mathrm{~Hz}, 2 \mathrm{H}), \delta 2.43(\mathrm{~s}, 3 \mathrm{H}), \delta 1.55(\mathrm{~m}, 2 \mathrm{H}), \delta$ $1.39(\mathrm{~d}, \mathrm{~J}=5.1 \mathrm{~Hz}, 3 \mathrm{H}), \delta 1.36(\mathrm{~m}, 2 \mathrm{H}), \delta 0.92(\mathrm{t}, \mathrm{J}=7.2 \mathrm{~Hz}, 3 \mathrm{H}) .{ }^{13} \mathrm{C}\left\{{ }^{1} \mathrm{H}\right\}$ NMR $(75$ $\left.\mathrm{MHz}, \mathrm{CDCl}_{3}\right) \delta 170.5,143.8,135.6,131.4,129.7,126.7,96.8,92.6,68.9,39.9,31.9$, 31.3, 21.3, 20.5, 18.9, 13.6. HRMS: $\mathrm{m} / \mathrm{z}(\mathrm{ESI})$ calculated $[\mathrm{MNa}]^{+}=392.1508$, measured $=392.1522$.<smiles>[10BH2]N(C=C)CCC(=O)OC(C)OCCCC</smiles>

\footnotetext{
${ }^{1}$ McKeon, J. E.; Fitton, P. Tetrahedron 1972, 28, 233-238.

${ }^{2}$ Kamath, S. S.; Uma, V.; Srivastava, T. S. Inorg. Chim. Acta 1989, 161, 49-56.

${ }^{3}$ El-Sharief, A. M. Sh.; Ammar, Y. A.; Zahran, M. A.; Ali, A. H.; El-Gaby, M. S. A. Molecules 2001, 6, 267-278.

${ }^{4}$ Giuliano, R. M.; Jordan, A. D., Jr.; Gauthier, A. D.; Hoogsteen, K. J. Org. Chem. 1993, 58, 4979-4988.

${ }^{5}$ Pak, C. S.; Kim, T. H.; Ha, S. J. J. Org. Chem. 1998, 63, 10006-10010.

${ }^{6}$ Gaulon, C.; Gizecki, P.; Dhal, R.; Dujardin, G. Synlett 2002, 6, 952-956.

${ }^{7}$ Gaulon, C.; Dhal, R.; Dujardin, G. Synthesis 2003, 14, 2269-2272.

${ }^{8}$ http://www.sigmaaldrich.com.

${ }^{9}$ Stacey, F. W.; Sauer, J. C.; McKusick, B. C. J. Am. Chem. Soc. 1959, 81, 987-992.

${ }^{10}$ Brown, H. C.; Wetzel, C. R. J. Org. Chem. 1965, 30, 3729-3733.
} 


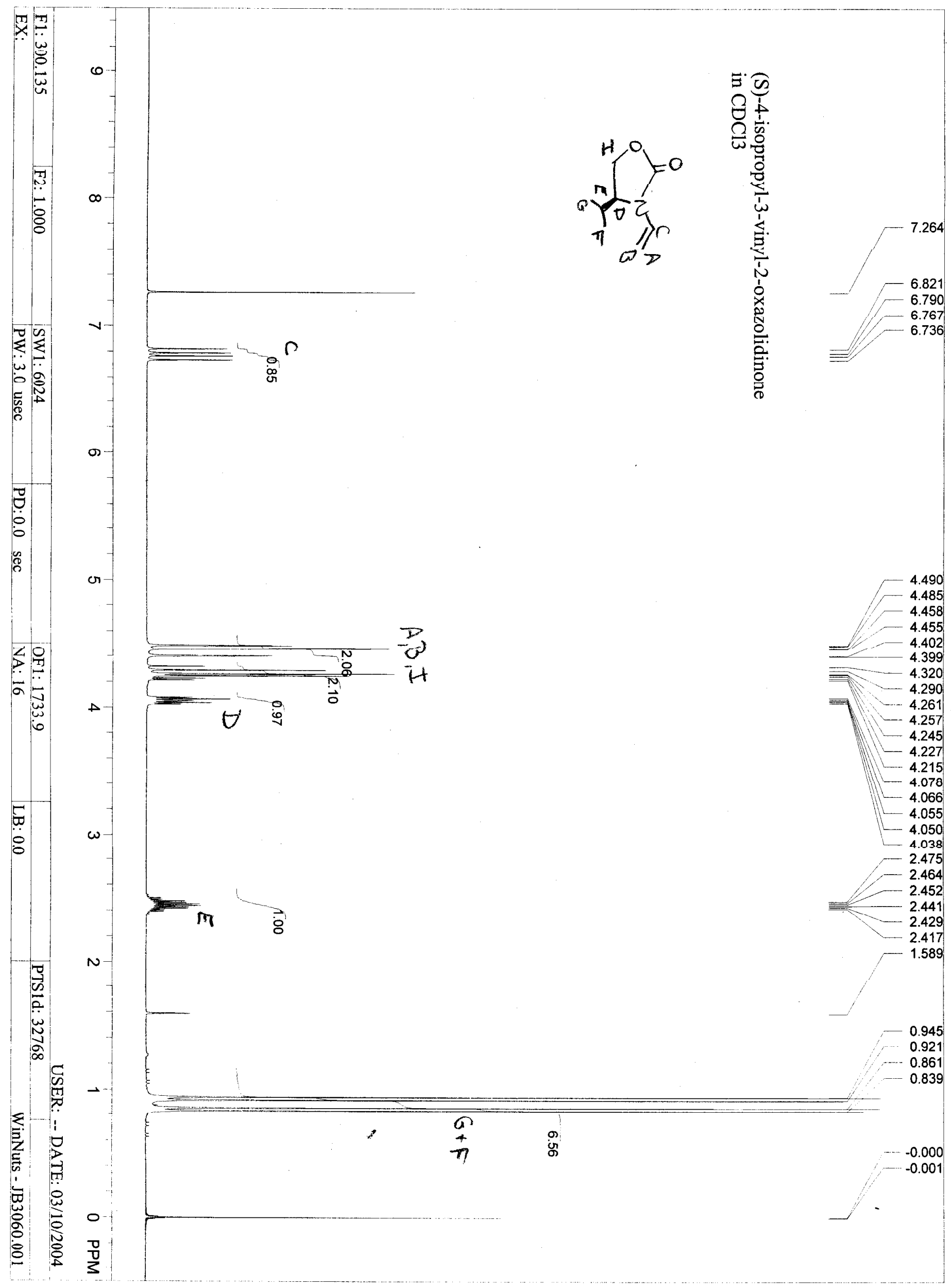




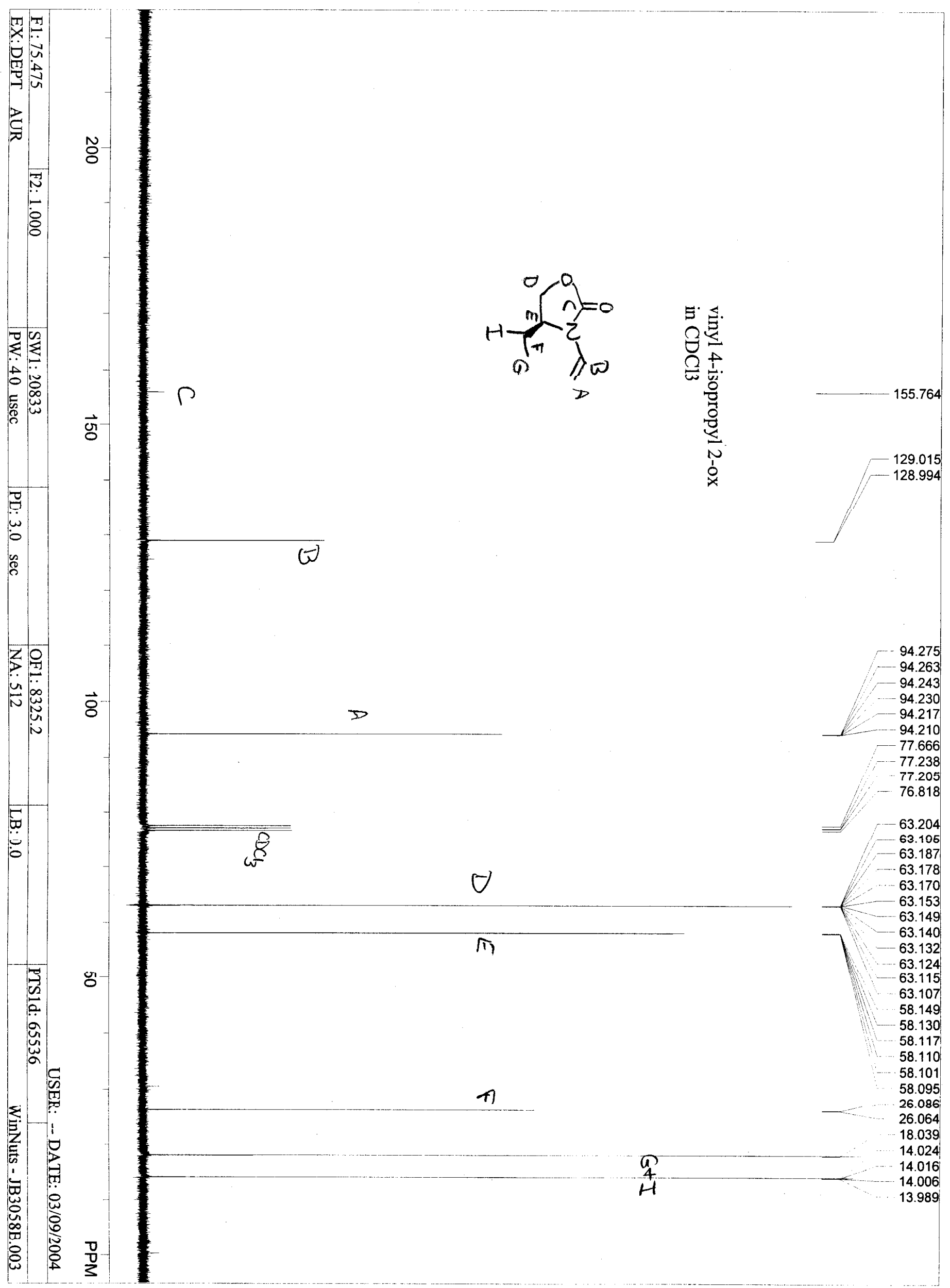




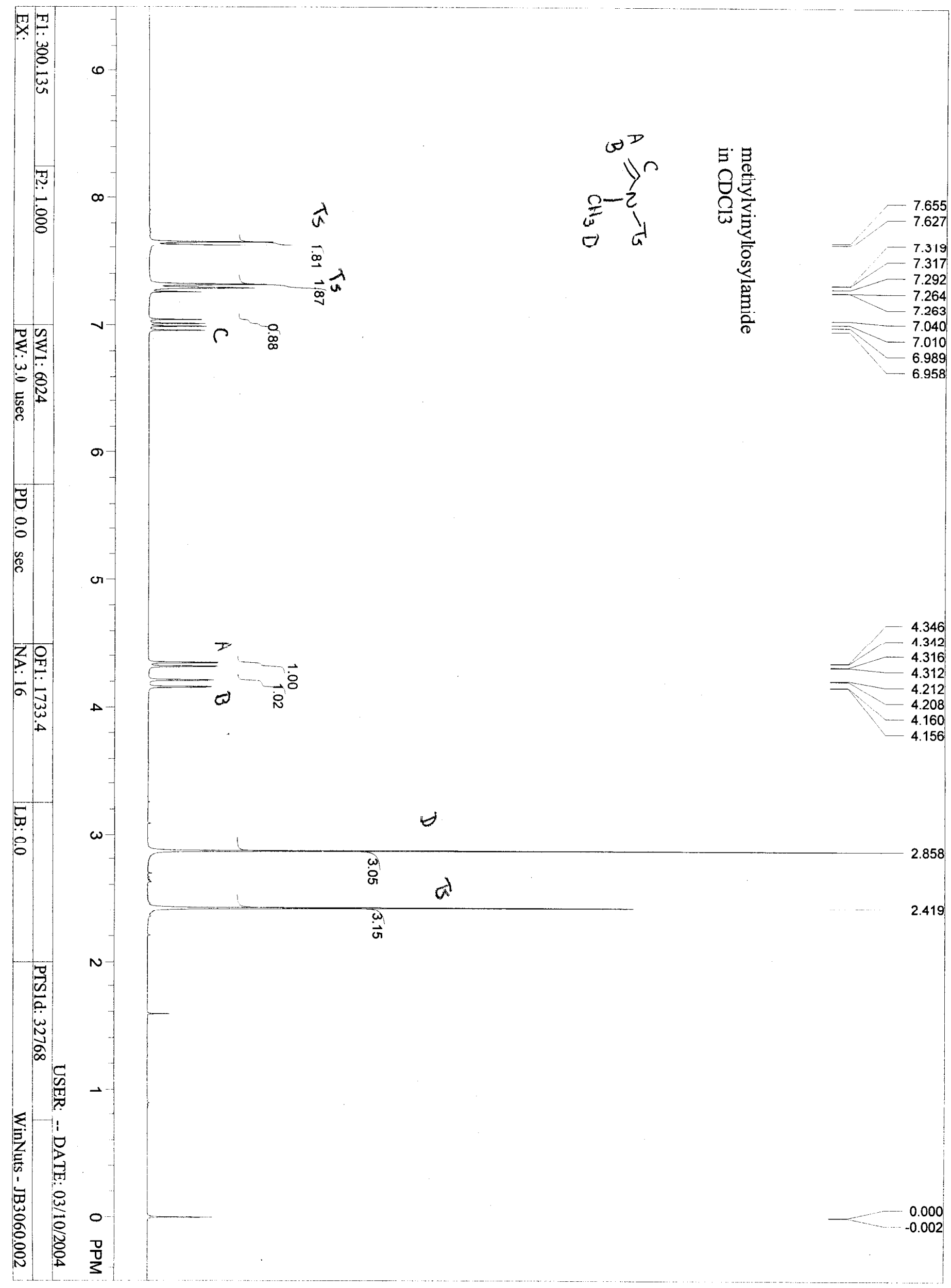




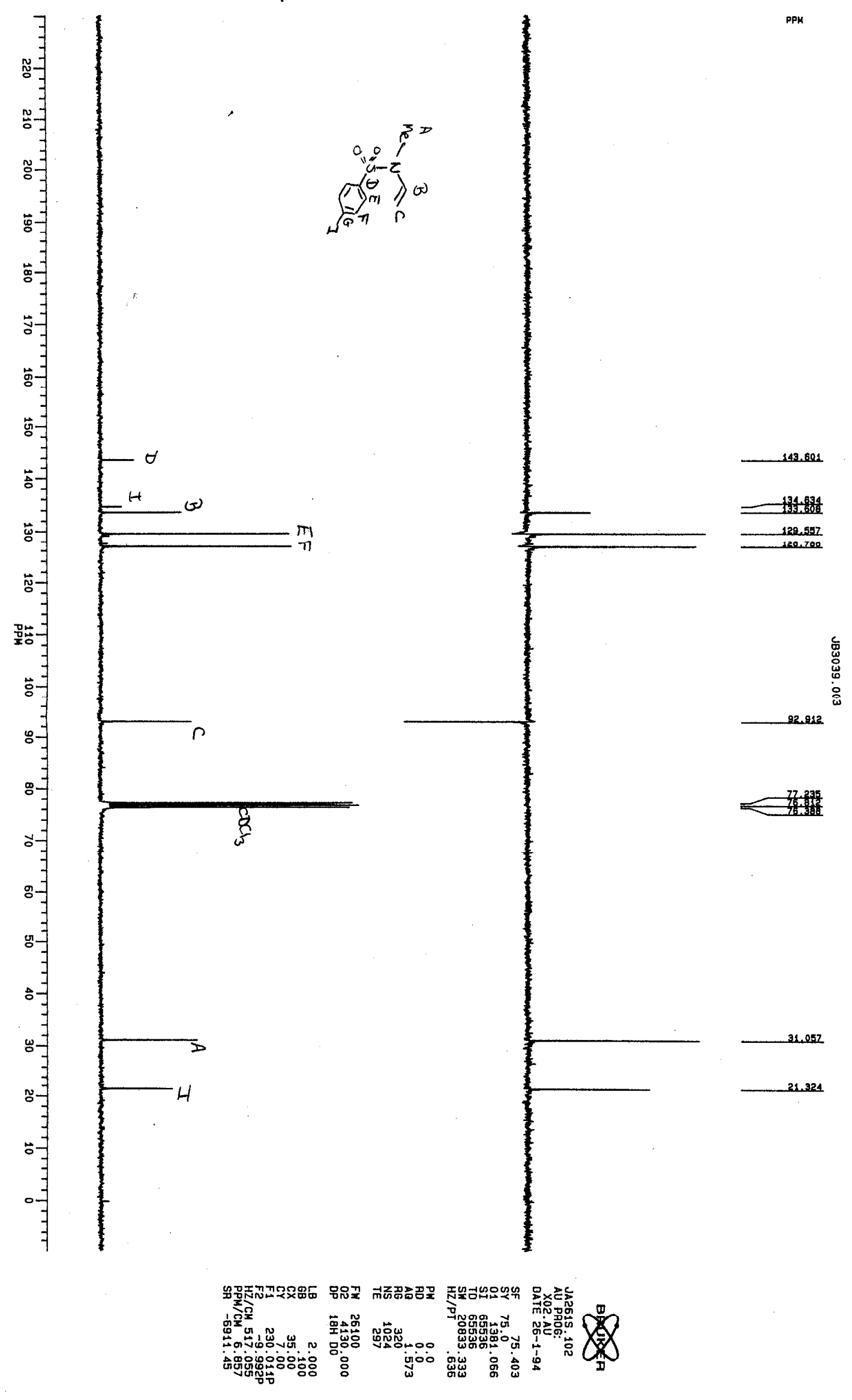




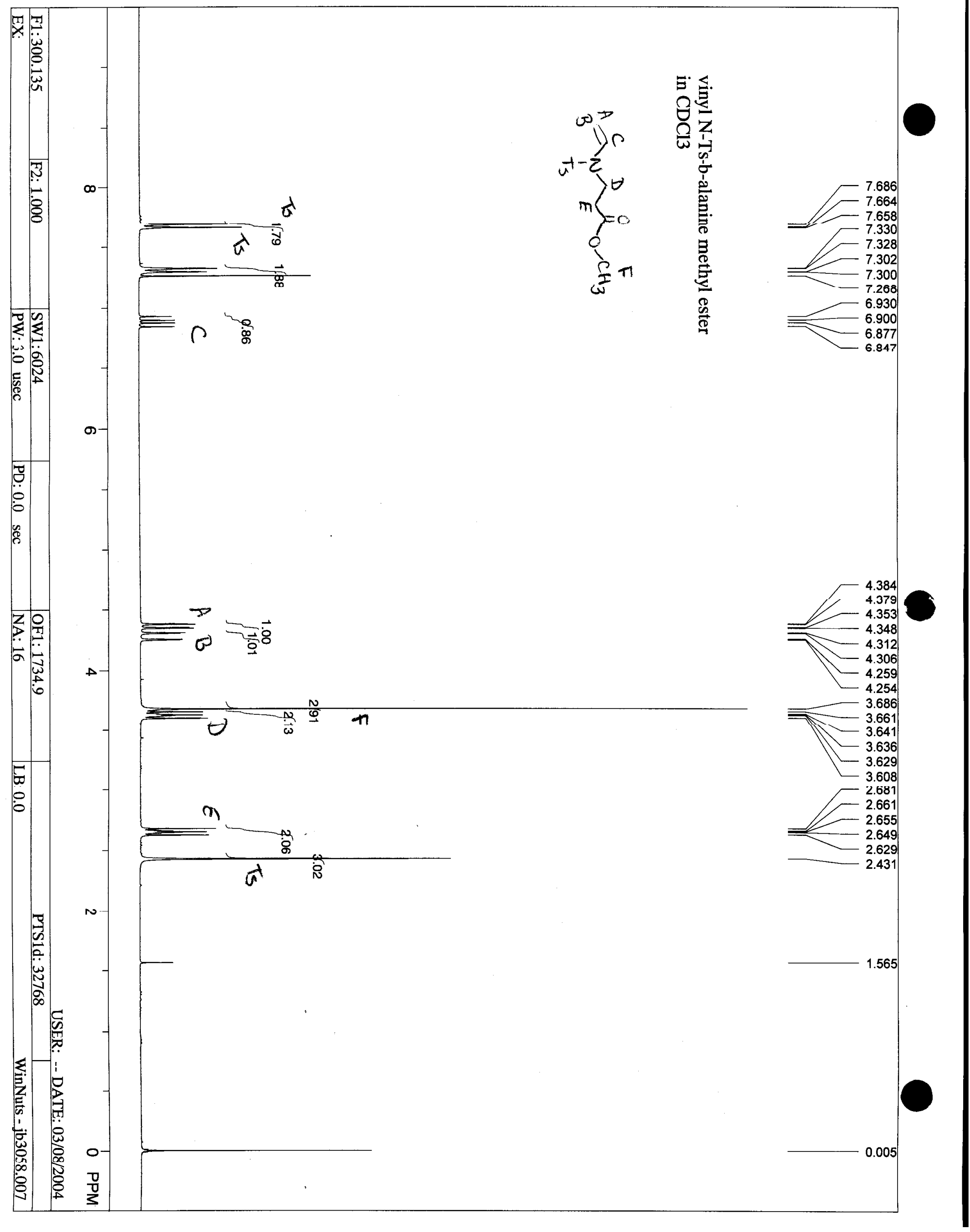




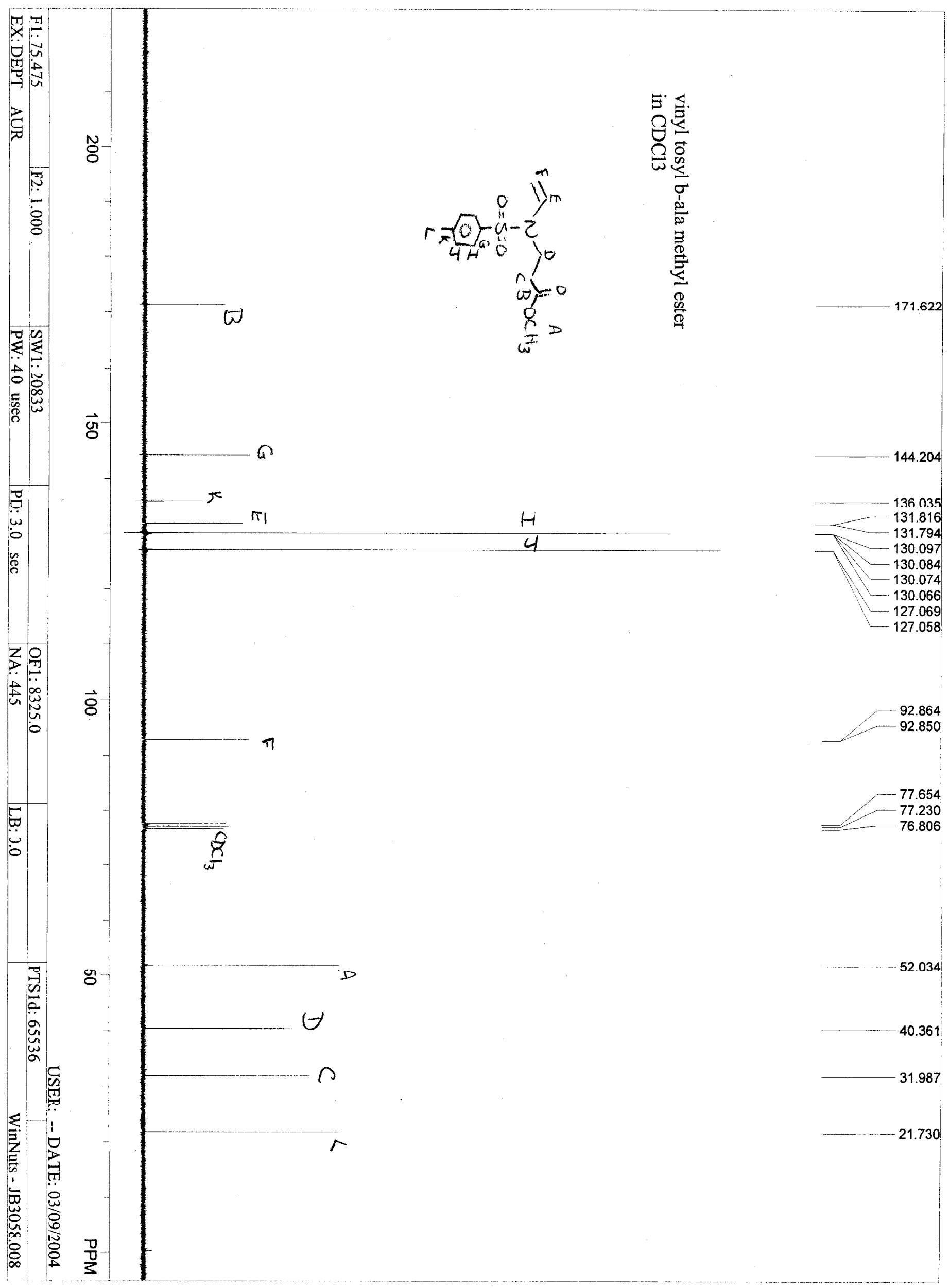




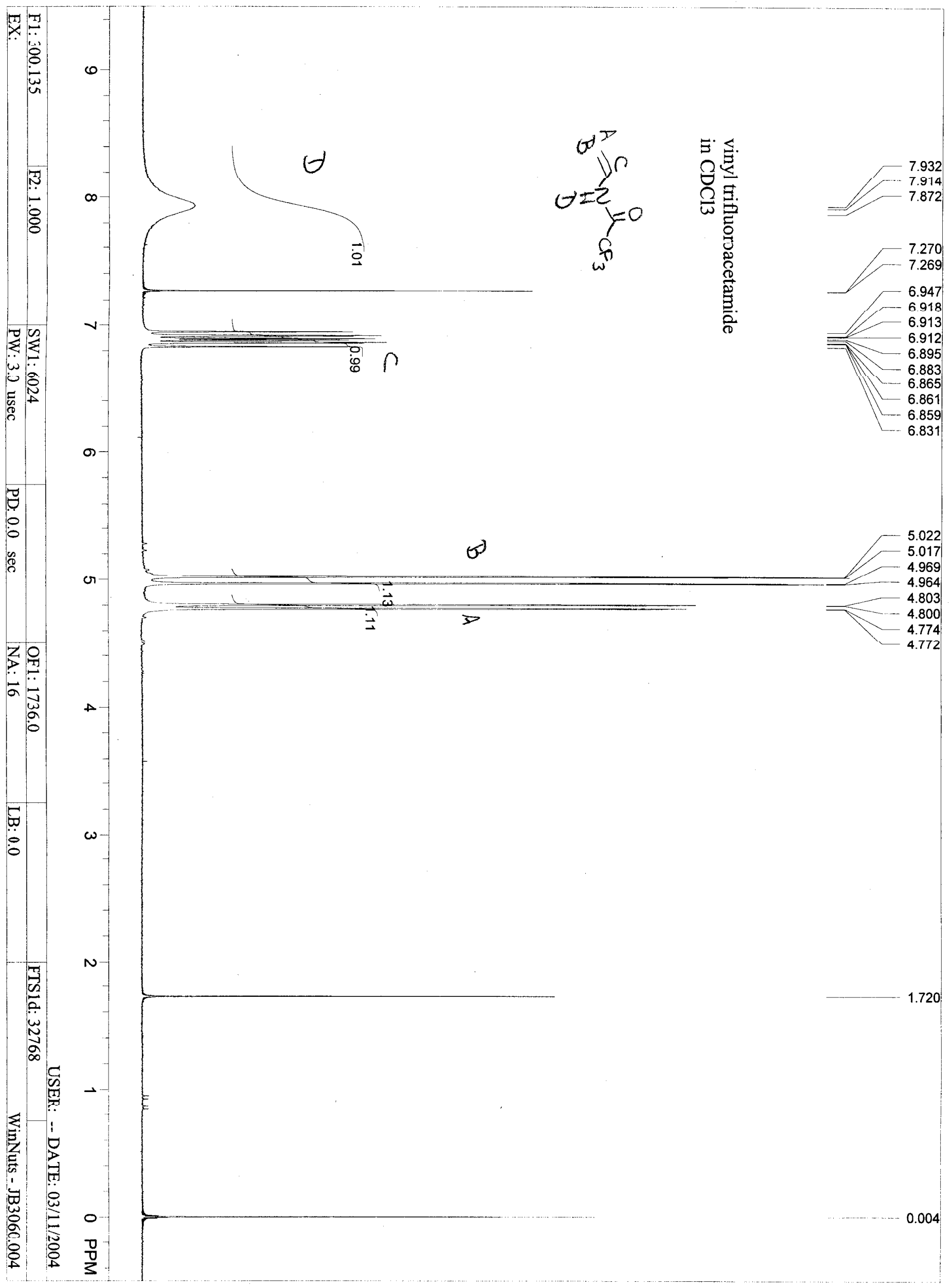




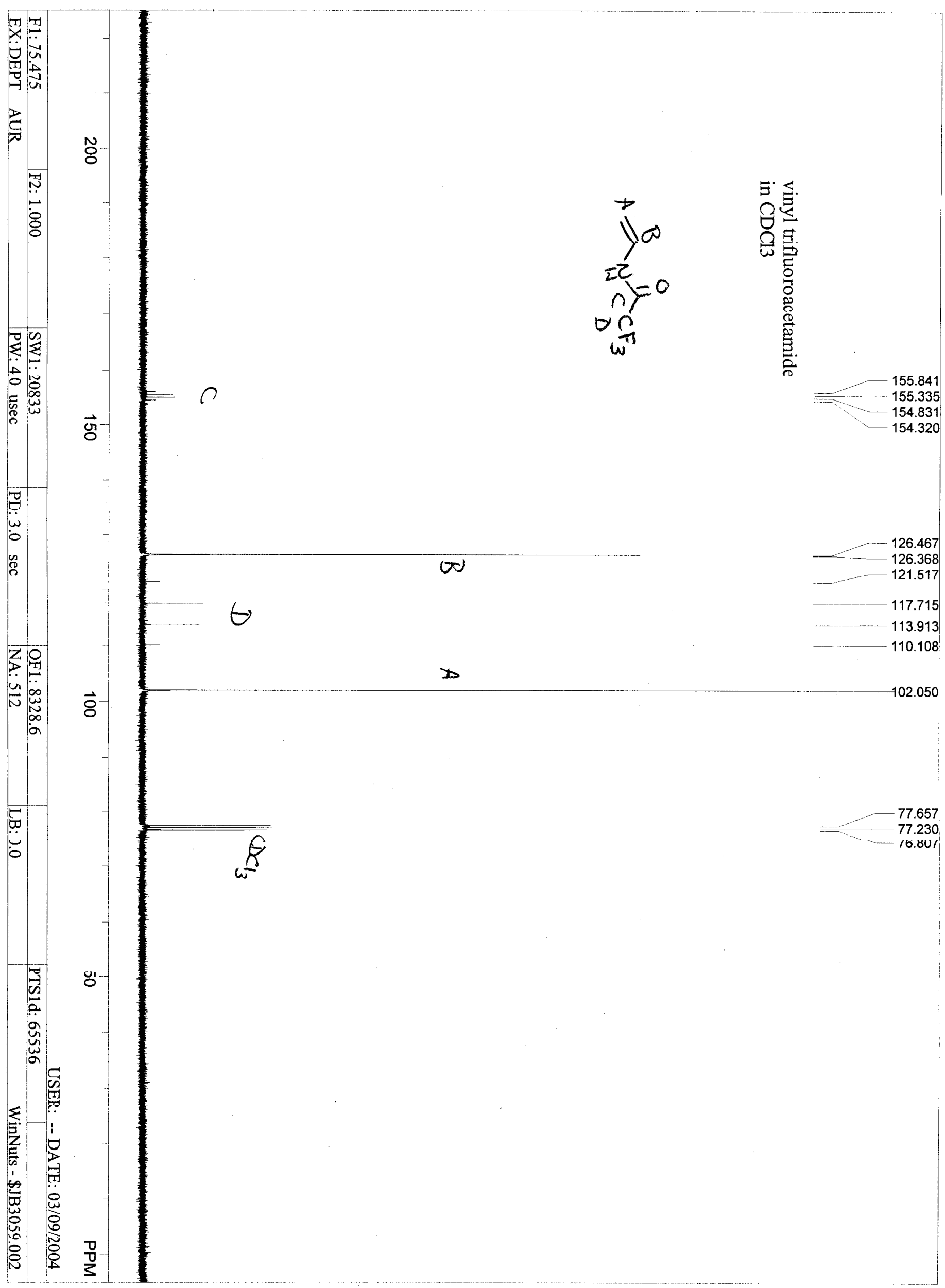




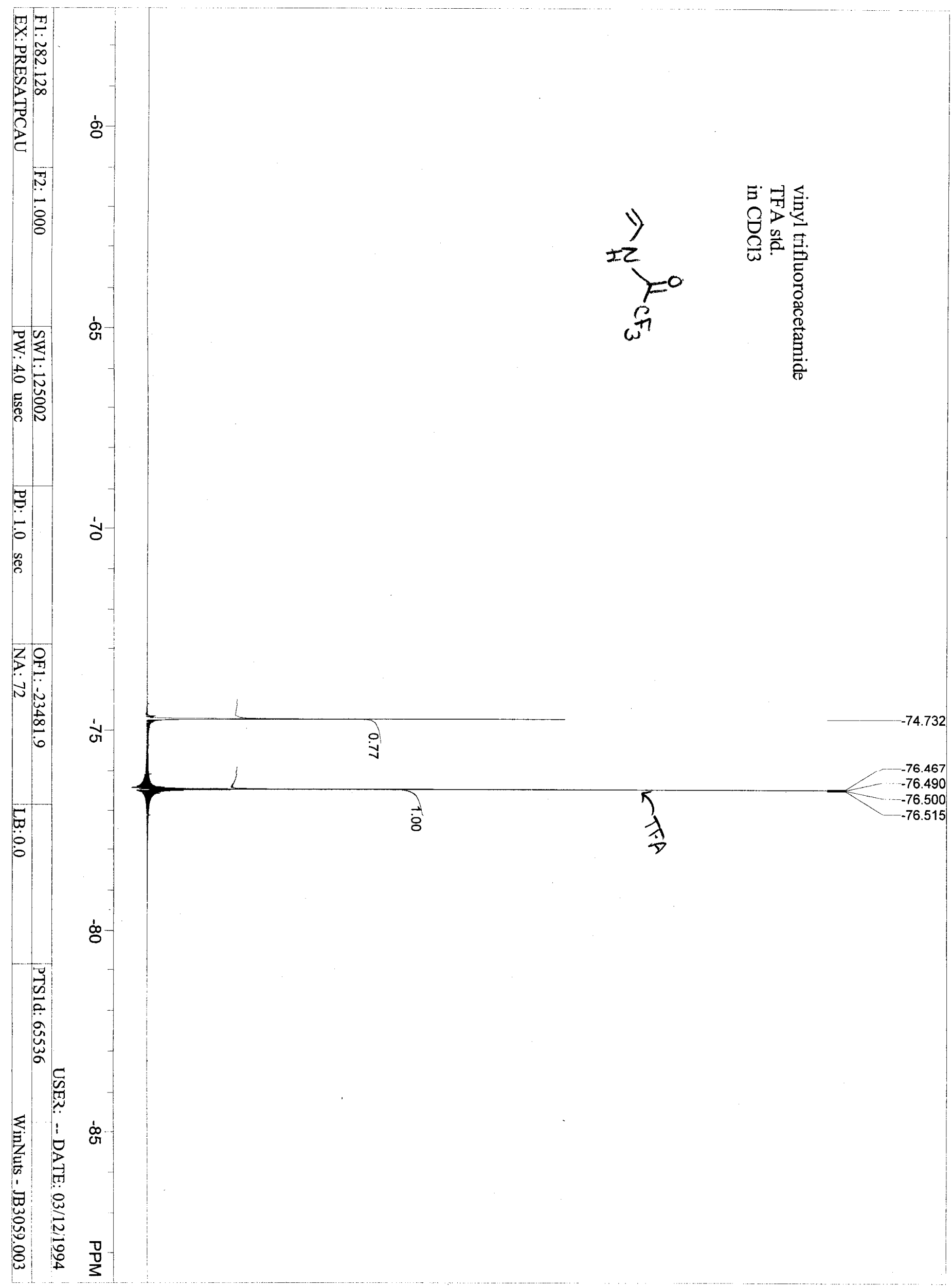




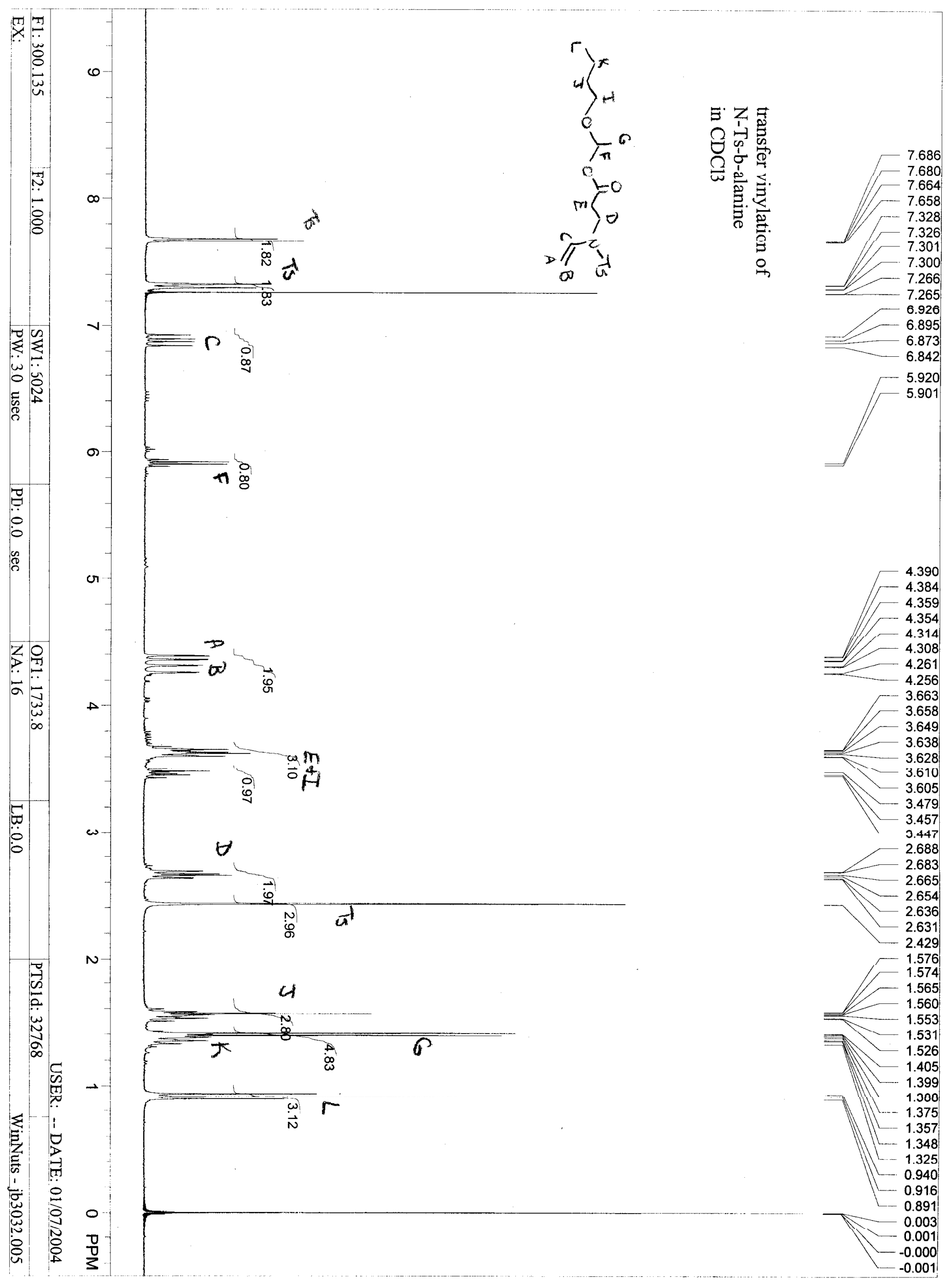




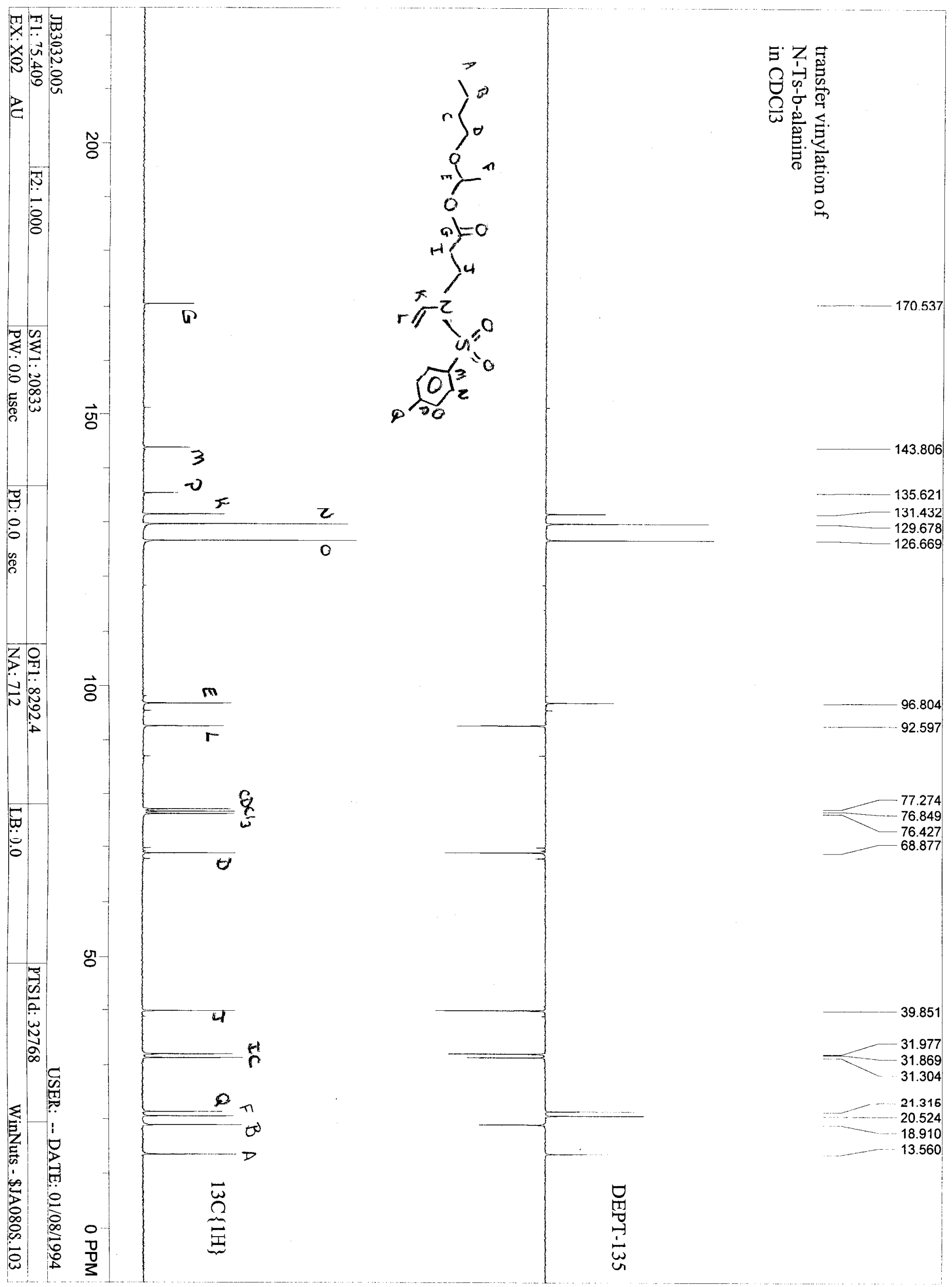

